\title{
Possibilities of the Lotka-Volter's Model using in the Area of Environmentalism
}

\author{
Gabriel Fedorko, ${ }^{1, *}$, and Vieroslav Molnár ${ }^{2}$ \\ ${ }^{1}$ TU Košice F BERG, Logistics Department, Park Komenského 14, 04001 Košice, Slovakia \\ ${ }^{2}$ TU Kosice, Faculty of Manufacturing Technologies with seat in Prešov, Bayerova 1, 08001, \\ Slovakia
}

\begin{abstract}
Environmental problems are becoming more and more important in all industrial sectors. This trend is closely linked to the increase of the number and extent of solved tasks and problems. Often these are complex problems that involve the impact of a wide range of different factors. Traditional conventional methods are not suitable for their solutions. Contrary, the use of simulation and optimization tools is very effective. Their application is based on attested formulas and models, for example, the model of Lotka-Volter. It is a continuous model of system dynamics, which is used successfully in various areas. Within the frame of environmentalism, it could find application in suitable simulation tools in the questions of a solution of the problem of water and water courses protection.
\end{abstract}

\section{Introduction}

Research of environment is a science that applies the knowledge of ecology. It examines human action on ecosystems, which deals with the prevention of environmental pollution, the repair of damage and prevention of undesirable interventions to the environment. For this reason, it has to make use of the knowledge from different disciplines. Environmental issues are interfering with the various areas of human activity, including industry [1]. Within the frame of environmental problems solution, it solves also problems related to production and used technologies [2]. Another broad area that environmentalism concerns are the processing of different types of wastes [3]. This discipline takes concerns in water and water course protection [4].

The area of water and water course protection presents the area with increased attention. But, this is a very demanding area and its solution uses methods, knowledge, and information that simplify and help to understand researched states and processes [5]. The method of computer simulation belongs to the methods with high potential for the area environmentalism. This method enables to acquire effective information, understand processes, and develop the solved problem from the environmental point of view.

\footnotetext{
* Corresponding author: gabriel.fedorko@tuke.sk
} 
The method of computer simulation offers for the solution of environmental problems related to water using of verified procedures, methods, and tools. These are presented, for example, by Celluar Automat [6] or the model of Lotka - Voltera.

\section{Theoretical scopes of the model Lotka-Voltera for the area of environmental problems solution}

The model of Lotka-Volter was created during 1925-1926 and it belongs to simple models that describe the relation of two separate, interdependent groups, components. Its deduction was realized for the need of population dynamics but over the years several modifications were created and it was successfully applied to various fields of science.

For the application of this model in the environmental area, we assume the existence of two components in direct interaction.

One component will be presented by a set of $x$ polluting particles, the number of which will grow exponentially, i.e. $\mathrm{x}=\mathrm{x}(\mathrm{t})$ is the size of the set of pollutants at time $\mathrm{t}$ and it applies (1).

$$
\frac{d x}{d t}=a_{1} x
$$

where $\quad \mathrm{x}-$ is a rate of pollutants

$\mathrm{a}_{1}-$ is a factor of pollutants increase

The second component will be a set y removing polluting particles $\mathrm{x}$. The size of the set $\mathrm{y}$ will gradually increase exponentially over time, so $\mathrm{y}=\mathrm{y}(\mathrm{t})$ is the size of pollutants disposal over time using cleaning system and it applies (2).

$$
\frac{d y}{d t}=-b_{2} y
$$

where $\quad y-$ is the initial performance of the cleaning system

$\mathrm{b}_{2}-$ is a factor in the cleaning system performance increase

Furthermore, it is needed to mention, that the set y removes only elements of the set $\mathrm{x}$. The rate of mutual interaction of both sets $\mathrm{x}$ and $\mathrm{y}$ is proportional to their size. The fixed ration of this action leads to the removal of the set $\mathrm{x}$.

On the basis of these assumptions, it is possible to state that the increase of the number of pollutants is proportional to the size of the set $y$ that ensures the removal of pollutants (3).

$$
\frac{d x}{d t}=a_{1} x
$$

where $\quad b_{1}-$ is the coefficient of predation of the set of pollutants $\mathrm{x}$ by the set $\mathrm{y}$ that eliminates them.

Conversely, the size of the set $\mathrm{y}$ is directly proportional to the size of the set of pollutants $\mathrm{x}$ and it applies (4). 


$$
\frac{d y}{d t}=-b_{2} y+a_{2} y x=y\left(a_{2} x-b_{2}\right)
$$

where $\quad a_{2}-$ is the coefficient of the set $y$ increase that eliminates pollutants $\mathrm{x}$.

The estimation of coefficients b2 and a2 can be done by linear regression. Determination of the coefficient of pollutants al increase can be realized on the base of results of measurements for a certain period of time. The value of the coefficient b1 is determined on the base of the efficiency of the cleaning process.

\section{Application of the Lotka-Volter model by computer simulation for the area of environmental problems solution}

Based on this information the use of the Lotka-Volter model for the area of environmental problem study was within the frame of performance monitoring and lifetime of filtration system that was installed at the discharge end of the sludge tank.

The filter was designed to work in three-phase mode. The first phase was a start-up, when the filter did not initially work at full power, but it gradually started running, until the second phase was reached. The filter worked on the full projected power, and after it passed, it went smoothly until the third phase. Here its performance gradually declined to the point where it was already adequate for the operating conditions and hat do be replaced. However, it is necessary to prepare in time, and therefore it is necessary to know the approximate duration of the exchange. The approximate time of exchange is necessary to estimate and know for the planning of operation and maintenance of the entire filtering device. For determination of the approximate time of the exchange, it was used the simulation software and the Lotka-Volter model.

Data about the initial volume of the contaminated fluid were input parameters for a creation of the calculation model and also information about the size of the inflow of the contaminated fluid in the sludge tank. Other data were information about the initial performance of the filter and phase of its change. Additional data were information about a change of filter performance on the base of the operating time and volume of the filtered fluid.

The simulation model was created as continuous because the activity of the modeled process had a continuous character. Therefore, it was necessary to select a suitable simulation software that would allow the creation of a continuous simulation model. For this reason, the program ExtendSim 8 was used. Blocks Value and Plotter (Fig. 1) from basic libraries of the program ExtendSim 8 were used for this model.

The simulation model contains four types of basic blocks of the program ExtendSim. These are blocks of Math, Constant, Lookup Table and Holding Tank. A functional and verified continuous simulation model was created by their combination. Its control and input of parameters for simulation calculations is designed using elements of the type Slider. Presentation of results is realized by the block I/O Plotter and the element type Meter. On the base of the analysis of the whole problem, it was decided that the simulation calculation will be deterministic and be realized by the fixed time step method. The simulation model is created by two separate branches representing the contaminated fluid and the filtration system. In terms of system dynamics, it comes from two separate systems that interact with each other. Especially, the filtration system by the washing of fluid reduces its volume in the sludge tank and repumpes the lifetime of the filtering device. 


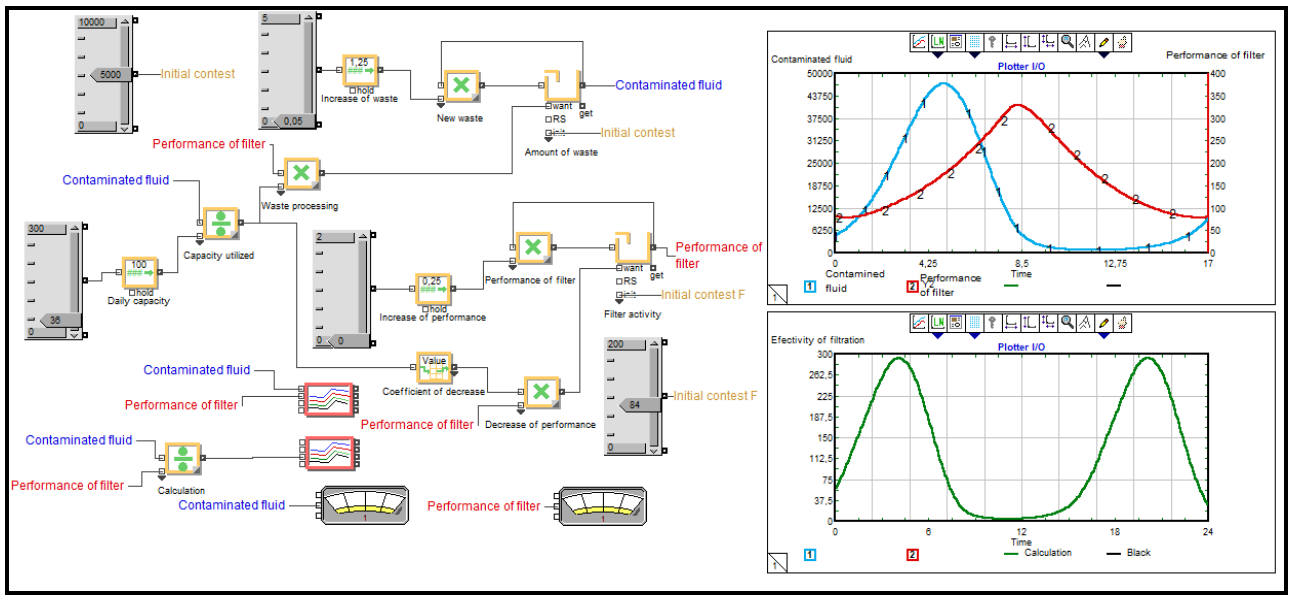

Fig. 1. Simulation model Lotka-Voltera in case of environmental problems. Source: Autors

\section{Results and Discussion}

A series of simulation calculations were realized with the model, the result is presented in the Fig. 2. From the result, it is possible to monitor the course of simultaneous processes of reduction and fluctuation of the volume of fluid in the blow-off tank and reduction of the performance of filtering device until the time when its performance is not adequate and its exchange is necessary. Due to the simulation, it was possible to determine the time of the filtration device replacement.

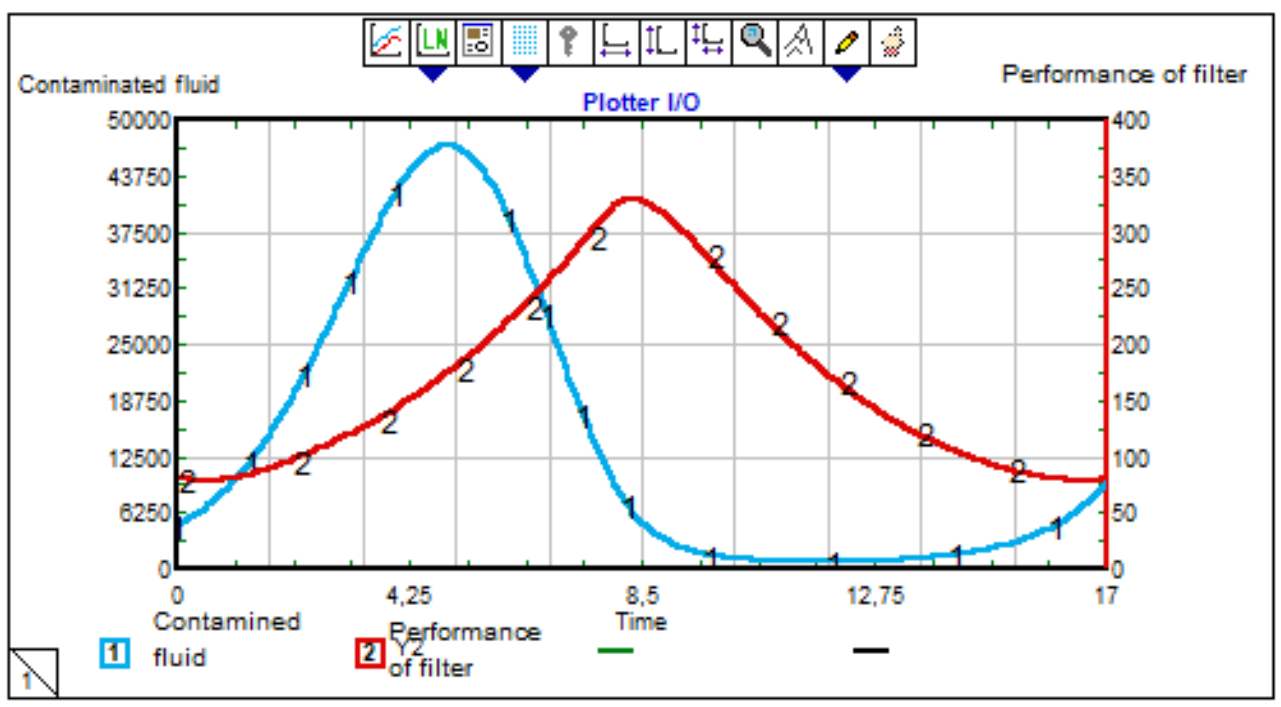

Fig. 2. Sample of sealing system of the conveyor belt. Source: Authors

\section{Conclusion}

The model of Lotka-Volter can be described as beneficial and applicable for the area of environmental studies. Environmental studies include many problem areas and tasks where 
the use of this model can be found. Combined with the method of computer simulation, the model can be effectively used for the solution of a wide range of problems connected with the environment.

The method of computer simulation presents an effective and powerful predictive tool for the area of the environment. Its application is possible in areas of air pollution, or in others, where it may be advantageous as necessary to predict future development and research the impact of different measures on relevant environmental processes. Main text paragraph.

The present paper is a part of research grant projects VEGA 1/0063/16, VEGA 1/0403/18, KEGA 018TUKE-4/2016.

\section{References}

1. F. Comunello, L. D. Trindade, M. F. Deimling, Rev. Gest. Amb. Sust. 6, 20 (2017)

2. L. Sobotova, M. Badida, Adv. Scie. Tech. 10, 6 (2016)

3. Y. Nuhoglu, E. Malkoc, Bio. Tech. 100, 6 (2009)

4. H. Q. Ni, , 29 th. A. Congr. I.A H. R. Proc. 29, 9 (2001)

5. T. S. Sun, J. Cui, I. C. Sust. E. E. Prot. Proc. 1, 11 (2015)

6. L. Nolle, H. Thormahlen, H. Musa, 30 th. E. Con. M. S. Proc. 30, 5 (2016) 